\title{
Mass Outflows in Narrow-Line Seyfert 1 Galaxies
}

\section{Michael Crenshaw ${ }^{1}$}

Georgia State University

Department of Physics and Astronomy, One Park Place South, SE, Suite 700, Atlanta, GA 30303 USA

E-mail: crenshawechara.gsu.edu

\section{Travis C. Fischer}

Georgia State University

Atlanta, GA USA

E-mail: crenshaw@chara.gsu.edu

\section{Steven B. Kraemer}

The Catholic University of America

Washington, DC USA

E-mail: steven.b.kraemerenasa.gov

\section{Henrique R. Schmitt}

Naval Research Laboratory

Washington, DC USA

E-mail: schmitt.henrique@gmail.com

We compare the characteristics of mass outflows of ionized gas in NLS1s with those in their broad-line counterparts. Are there differences that yield clues to the geometry of the outflows or the driving mechanisms? We address these questions using HST high signal-to-noise, high resolution spectra of the UV absorbers at distances of 0.1 to 10 parsecs from the central nucleus and long-slit spectra of the emission-line outflows on larger scales in their narrow-line regions.

Narrow-Line Seyfert 1 Galaxies and Their Place in the Universe (NLS1

Milan (Italy)

April 04-06, 2011

1 Speaker 


\section{Introduction}

We have observed several narrow-line Seyfert 1 (NLS1) galaxies in the course of obtaining UV spectra of nearby AGN at high spectral resolution $(\mathrm{R}=15,000-40,000)$ with STIS and COS, and optical long-slit spectra of these same AGN with STIS at high angular ( $\sim .1$ ") resolution. The questions we would like to address are as follows. 1) What are the outflow properties of NLS1s compared to broad-line Seyfert 1s (BLS1s)? 2) Are NLS1s viewed at a special angle (e.g., pole-on)? We note that NLR kinematics are probably the best way to determine the inclinations of individual AGN. To address these questions, we use HST observations of UV absorbers at distances of 0.1 to $\sim 10$ parsec from the central nuclei and NLR outflows at distances of 10 to $1000 \mathrm{pc}$.

\section{UV Absorbers}

Our best UV spectrum of an NLS1 is the COS spectrum of NGC 4051 obtained on 2009 December 11, which is shown in black in Figure 1 [1]. We overplot the STIS spectrum from 2000 March 24/25 in red. Given the differences in signal-to-noise and spectral resolution, we see no evidence for changes in any of the absorption components over $\sim 10$ years. The absorption spectrum is similar to that of the BLS1 NGC 4151 [2], in that there are numerous outflowing components, many of which are broad and competely saturated, indicating large column densities. In the case of NGC 4151, we have suggested that the high-column absorption is due to a line of sight that is at a polar angle of $\sim 45^{\circ}$ with respect to the accretion-disk axis [3].

In Figure 2, we plot the radial velocity centroids and full-width at half-maxima (FWHM) for all of the individual absorption components in Seyfert galaxies observed at spectral resolutions $>10,000$ in the UV with HST and far-UV with FUSE [4]. There is no correlation between the two parameters, and it appears that NLS1s and BLS1s cover the same parameter space. Thus, there is no evidence that NLS1s differ from BLS1s in terms of absorption kinematics.

\section{NLR Outflows}

We have obtained HST long-slit spectra of the [O III] emission in the NLR of NGC 4051 from the Multimission Archives at Space Telescope Science Institute. A detailed analysis of these data is given by T. Fischer in these proceedings [5]. As shown in Figure 3, the axis of NLR bicone is only $\sim 12^{\circ}$ from our line of sight. Thus, it appears we have a nearly pole-on view of this NLS1, which could offer a possible explanation for the relatively "narrow" broad lines if we assume that the BLR has a strong rotational component. However, we need to model the kinematics of the NLRs in a number of NLS1s and BLS1s to fully test the idea that NLS1s are viewed pole-on. 


\section{A Schematic Outflows Model}

Given the small outer opening angle of the bicone in NGC 4051, Figure 3 shows that we also have a view into the bicone that is close to the outer edge. This may explain the highcolumn UV absorption that we see in the UV, similar to that seen in NGC 4151. Although we have inclinations for only a few Seyfert $1 \mathrm{~s}$ based on their NLR kinematics, we are starting to see a trend, as shown in Figure 4. Based on these few AGN, it appears that the column density of the absorbers is increasing with polar (viewing) angle (note that the bicone should be narrower for NGC 4051 - this is just a schematic model). As we continue to determine inclinations of AGN based on their NLR kinematics, we can determine what our view is like if we are looking right down the bicone. Given the above trend, we might be viewing unabsorbed Seyfert galaxies from this direction. On the other hand, maybe we are seeing NLS1s in the pole-on view. It is difficult to accommodate both cases, because many NLS1s show intrinsic absorption, unless most NLS1s have narrow (small opening angle) bicones. We are currently analyzing a dataset of $\sim 30$ Seyfert galaxies to address these issues.

\section{A Schematic Outflows Model}

In conclusion, it appears the UV absorber outflows are similar in both NLS1s and BLS1s. NLR outflows are also similar and likely common in all Seyfert types (NLS1, BLS1, Seyfert 2). We have a view of the NLS1 NGC 4051 that is close to pole-on, but it is not yet clear if this is the case for NLS1s in general. Further work is needed to determine which AGN properties, such as total absorption column, vary with polar angle. 


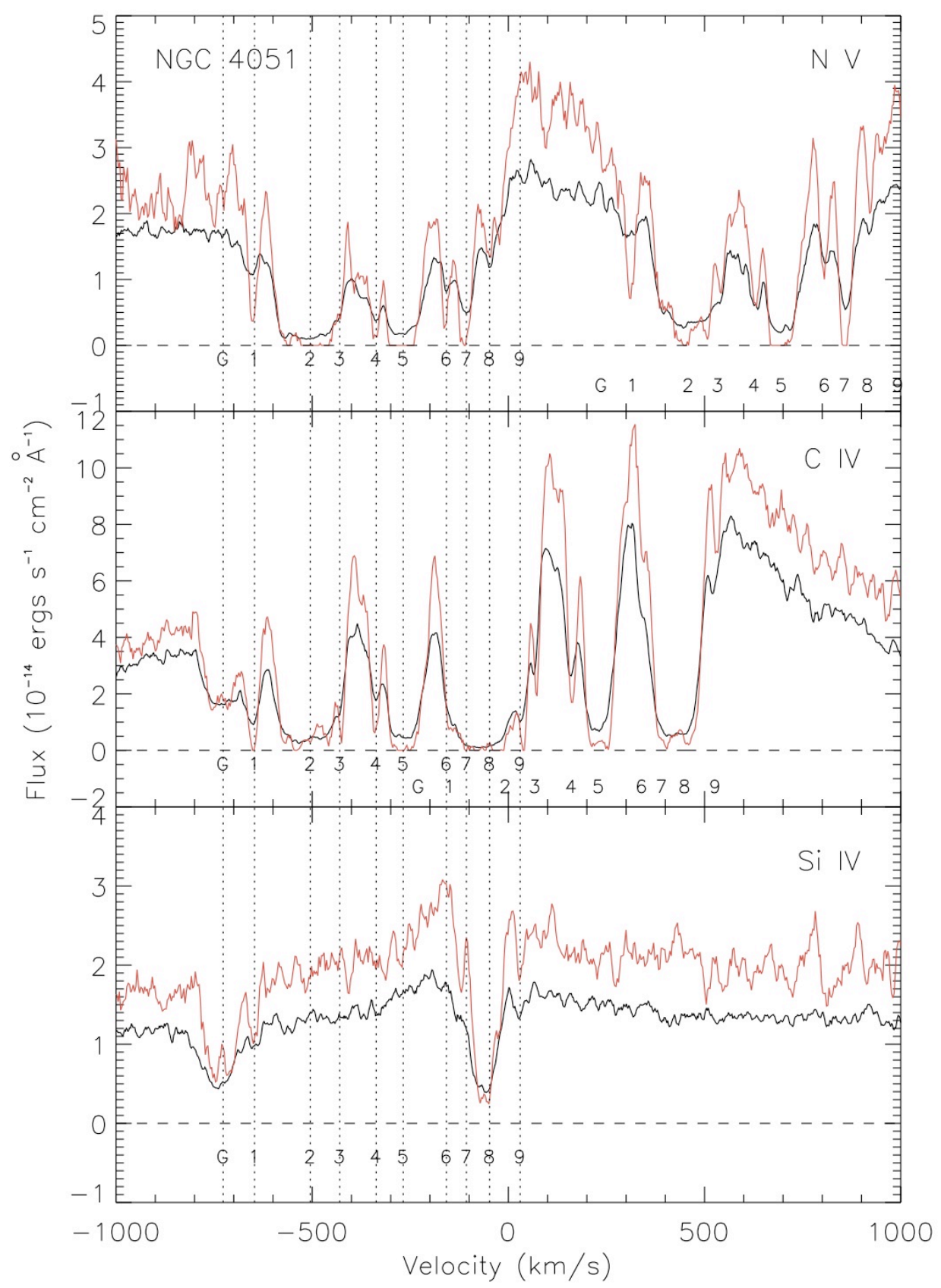

Figure 1. Kinematic components of absorption for high-ionization lines in the UV spectrum of NGC 4051. The black lines give the COS spectrum and the red line give the STIS spectrum. Fluxes are plotted as a function of radial velocity for the strongest member of each doublet, relative to an emission-line redshift of $z=0.002295$. 

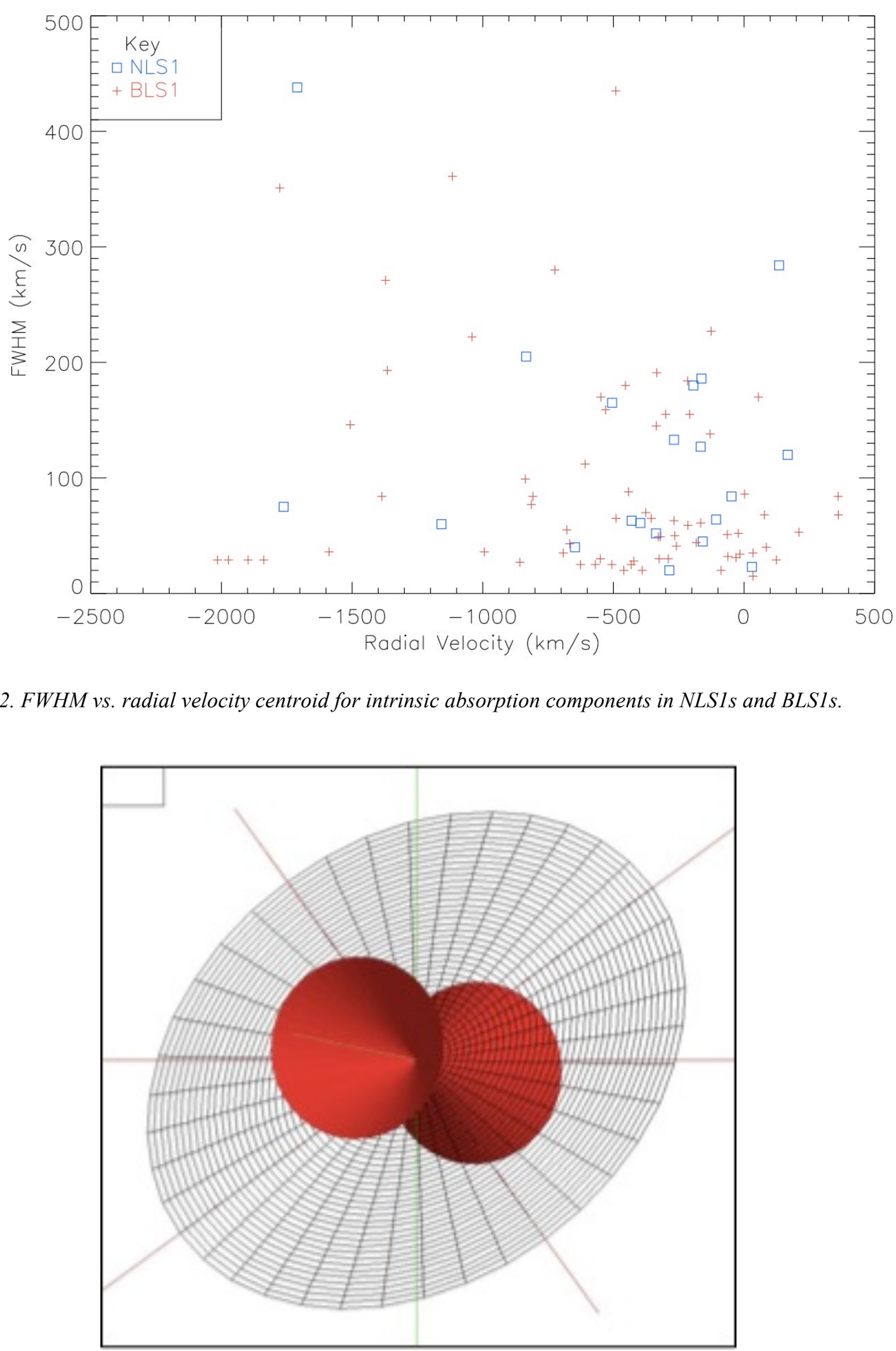

Figure 3. Geometric model of the NLR bicone and host galaxy in the NLS1 NGC 4051. 


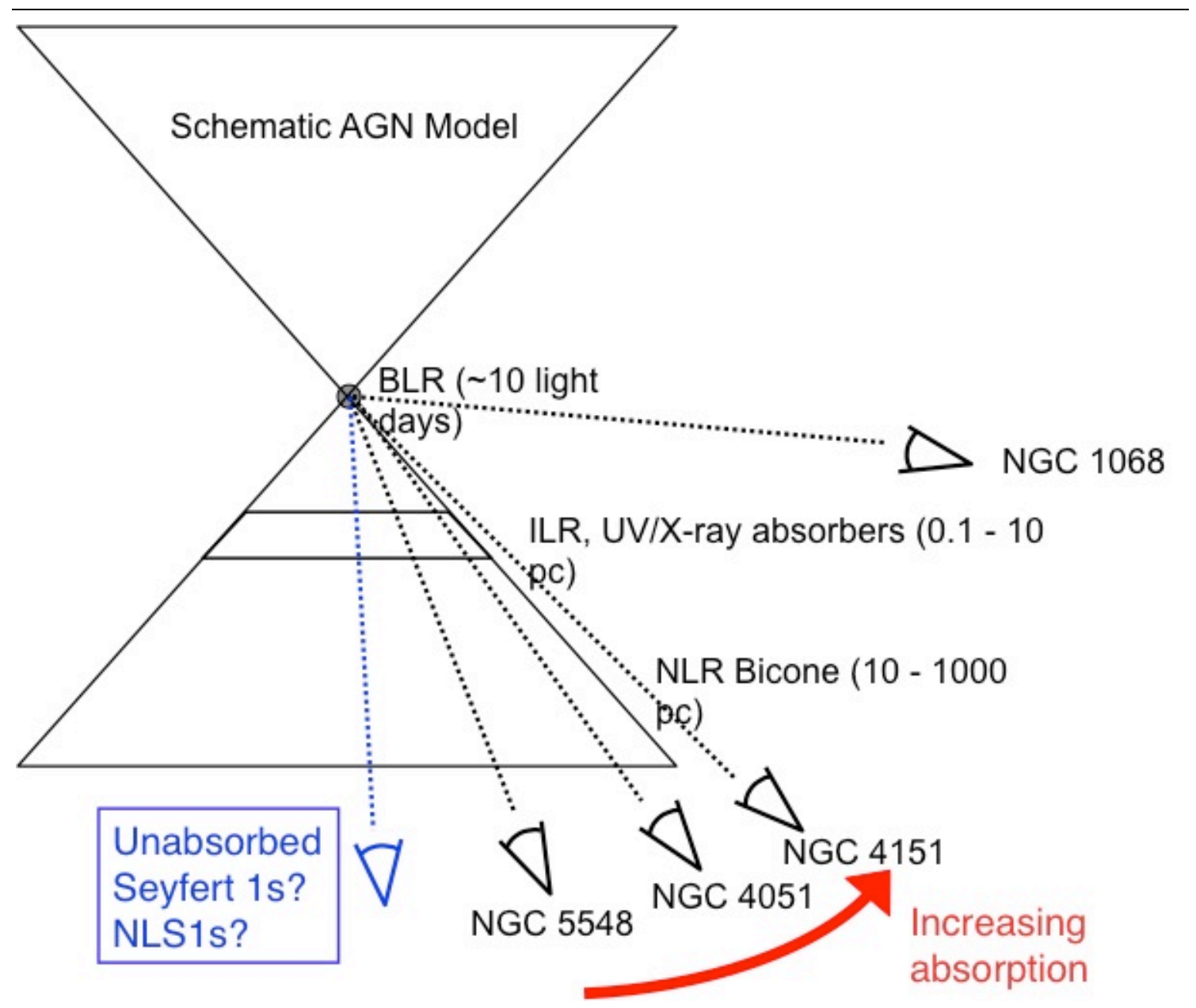

Figure 4. Schematic model of the structure of AGN outflows. The absorption column of ionized gas appears to increase with increasing polar angle. The Compton-thick Seyfert 2 galaxy NGC 1068 is shown for comparison, although in this case the absorption is likely due to cold neutral gas.

\section{References}

[1] Kraemer, S.B., et al. 2011, in preparation.

[2] Kraemer, S.B., et al. 2006, ApJS, 167, 161

[3] Crenshaw, D.M. \& Kraemer, S.B. 2007, ApJ, 659, 250.

[4] Dunn, J.P., et al. 2008, AJ, 134, 1061.

[5] Fischer, T.C., et al. 2011, this proceedings. 